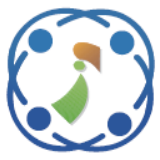

\title{
Rapid Frequent Pattern Growth and Possibilistic Fuzzy C-means Algorithms for Improving the User Profiling Personalized Web Page Recommendation System
}

\author{
Sipra Sahoo $^{1 *} \quad$ Bikram Kesari Ratha ${ }^{2}$ \\ ${ }^{I}$ Department of Computer Science \& Engineering, Siksha 'O' Anusandhan University, India \\ ${ }^{2}$ Department of Computer Science \& Engineering, Utkal University, India \\ * Corresponding author’s Email: sipraphd2017@gmail.com
}

\begin{abstract}
Web page recommendation systems are used to recommend the future web page views to World Wide Web (WWW) users. Existing personalized web page recommendation systems are still limited to several problems such as, cold start, sparse data structures (Sparsity), and no diversity in the set of recommended items (Content Overspecialization). In order to overcome these difficulties, we proposed a new personalized web recommendation system by considering the different contributions of the training samples (MSNBC dataset, and castings technology international dataset). At first, the web pages are converted into number of sequences. An effective clustering algorithm called as Possibilistic Fuzzy C-Means (PFCM) is employed to identify the neighbourhood of each user profile. Then the association rule mining technique, Rapid Frequent Pattern Growth (RFPG) algorithm is used for mining the frequent web pages. Finally, the experimental outcome shows that the proposed approach delivers the high priority of web pages and also recommends the related web pages. Finally, the experimental outcome shows that the proposed approach improved accuracy in web page recommendation up to 50-60\% compared to the existing methods.
\end{abstract}

Keywords: Frequent pattern growth, Fuzzy c-means, Rule mining Web page recommendation system, World wide web.

\section{Introduction}

Exceptional growth of an information on the WWW with the development of advanced electronic devices has made the web information increasingly important in almost everybody's life [1, 2]. People use the internet to get information on area of interest, do research related to work or study, get good deals for commodities or travel, increases the awareness of their surroundings, and to get the latest news, etc. [3]. In the recent days, huge amount of web pages, web sites and web documents are available, so the search engine returns the thousands of related links to a search query. The outline of the current websites has overwhelmed the web users by offering many choices [4]. Hence, the web users tend to make poor decisions when surfing the Web, due to an inability of managing the enormous amount of information. Also, it has become very difficult for users to get the relevant information [5]. In order to extract the useful patterns in web usage, an effective system is implemented named as a web-page personalization recommendation system. Web personalization system has become more popular as the users get a relevant links of various webpages and websites without searching repeatedly [6]. The existing personalized web-page recommendation systems have some complexities such as the cold start problem, which refers to the situation when a new user or item just enters into the system [7].

In such cases, it is really very difficult to provide recommendations as in the case of new users, there is a very less information about the user and also for a new item, no ratings are available [8, 9]. The personalized web recommendation is carried out based on the relations between the products in the previous transactions and also another possibility of recommendation is based on the results given by user about a product and similar feedbacks from the different users [10]. To overcome these problems, a 
new personalized web recommendation system is proposed. In this experimental research, the webpage recommendation system is performed on the reputed datasets: MSNBC dataset, and castings technology international dataset. After the acquisition of data, convert the web pages into number of sequences. The PFCM clustering technique is performed on the number of sequences for identifying the neighbourhood of each user profile. After identifying the user profiles, the association rule mining is applied using the RFPG algorithm for mining the frequent web pages. This proposed model provides the highest priority of web pages and also recommends the personalized web page to the user.

This paper is composed as follows. Section 2 survey several recent papers on the web-page recommendation system. In section 3, PFCM method is presented with RFPG methodology for recommending the related web pages. In Section 4, comparative analysis for proposed web-page recommendation system using MSNBC, and castings technology international datasets are presented. The conclusion is made in the Section 5.

\section{Literature review}

Several techniques are suggested by researchers in the web-page recommendation system. In this scenario, a brief evaluation of some important contributions to the existing literatures is presented.

T.T.S. Nguyen, H.Y. Lu, and J. Lu [11] presented the new models such as, ontology based model and conceptual prediction based model. An ontology model provides the relation between semantic network domains and web pages. The conceptual model predicts the next web page request of the users through querying knowledge bases and provide a semantic network of the web usage knowledge. This model was the combination of both domain knowledge and web usage knowledge. This information retrieval system handles the one by one user request. Whereas, multiple user request was difficult to handle in this system.

R. Geetharamani, P. Revathy, and S.G. Jacob [12] proposed a new model named as Apriori Prefix Tree (APT) algorithm for web page prediction. The objective of this literature was to predict the probable subsequent page in the web pages based on their navigating behaviour. APT algorithm was used to mine the common patterns, which were investigated based on the rule consequents and antecedents. In the higher user recommendation system, less user logs were received, so the performance of web prediction accuracy was decreased.
R. Katarya, and O.P. Verma [13] illustrated a new web-based recommender system, which was based on the sequential information about user's navigation on the web pages. In this literature, Fuzzy C Means (FCM) clustering technique was used to provide the recommender system sequentially. Using FCM, the Top-N clusters were achieved, which were further used for identifying the similar users for a target user. Then, calculate the weight for each page category and finally determine the accuracy of the system. This approach was not concentrated on the content of the web page and also FCM was not suitable for a new user, because it requires at least six web logs per user.

N.T.S.H. Rizvi, and R.R. Keole [14] presented an intelligent web recommendation system using a term pattern frequency mining technique for predicting the next web page. A desktop search utility uses the web usage mining process for finding the term patterns in web query data, which was used for predicting the possible next pages from browsing sessions in a very less time. The proposed framework recommends the best web pages based on the user queries using the google search engine. So, the web page recommendation for a multiple website at the same domain was required.

S.P. Malarvizhi, and B. Sathiyabhama [15] has presented a new methodology called as a $\mathrm{T}+$ weight tree algorithm for finding the frequent page-sets. The proposed $\mathrm{T}+$ weight tree algorithm finds the frequent page-sets based on the weights in a single scan of the database. The time elapsed for finding all the subsets of a set was more, so an effective process was required to reduce the time. The proposed methodology was much more beneficial to find the frequent page-sets. In the weighted association rule mining technique updating process not occurred, so it doesn't consider the behavioural pattern of web pages.

A.S. Tewari, and A.G. Barman, [16] proposed a recommendation system using content based collaborative filtering, association rules and opinion mining. Whereas, the content based collaborative filtering was used to monitor the user shopping behaviour. Also, the proposed methodology finds the like-minded people with the target user that may cooperate with each other in the form of items ratings using collaborative filtering. The proposed methodology utilizes association rule mining for the analysis of current market trend, which generates association rules only from those items that are liked by the users. In this literature, the proposed methodology utilizes own unique weight that summarizes the reviews and generates the weights for each item based on customers' reviews. These weights help in estimating the popularity of an item among customers. If there was not enough 
information to build a solid profile for a user, the recommendation could not be provided correctly.

V. Vellaichamy, and V. Kalimuthu, [17] developed a hybrid collaborative movie recommender system, which was the combination of both Fuzzy C Means clustering (FCM) and bat optimization technique. This combined methodology improves the recommendation quality. FCM clustering was utilized to cluster the users into different groups. On the other hand, bat algorithm was utilized to obtain the initial position of the clusters. At last, the proposed methodology creates the movie recommendation for target users. The major drawback of FCM was limited content analyser. If the content does not contain enough information to discriminate the items precisely, the recommendation was not precisely at the end.

To overcome the above mentioned drawbacks, an effective algorithms PFCM and RFPG is combined for improving the user profiling personalized web page recommendation system.

\section{Proposed methodology}

The proposed personalized web page recommendation system consists of five steps such as, data acquisition, pre-processing, similarity measure, clustering and pattern mining. A general block diagram of personalized web recommendation system is represented in Fig. 1. The brief description about the proposed methodology is determined below.

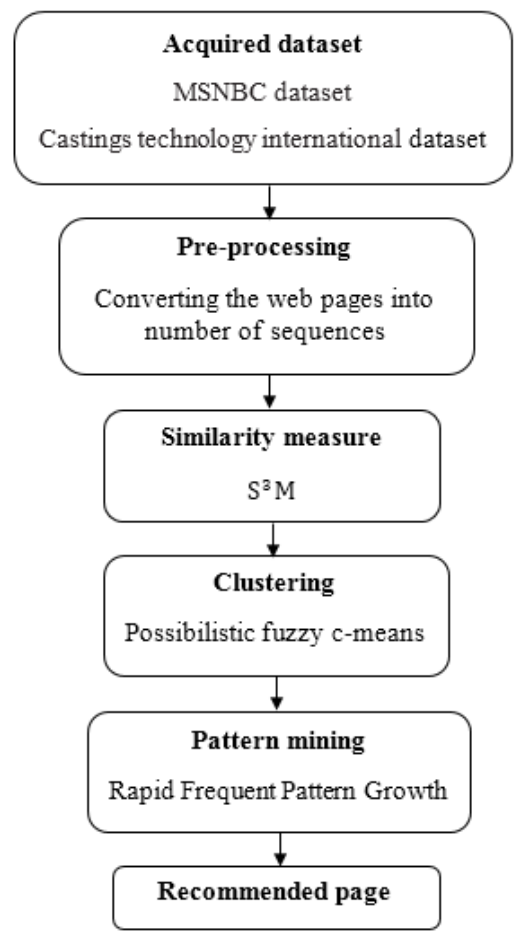

Figure.1 A General block diagram of the proposed approach

\subsection{Pre-processing}

In the initial stage of the personalized web page recommendation system, the web logs and web content of user data are taken from the standard benchmark datasets such as, MSNBC dataset and castings technology international dataset. In the preprocessing phase, the web logs data or web pages are converted into number of sequences and also identifies the user profiles. Then, all the similar sequences are clustered using PFCM with the help of S3M similarity measure.

The S3M similarity measure or set similarity measure considers the both content as well as the sequence of the input data for computing the similarity between the web users. The S3M contains two phases: one is set similarity that quantifies the composition of sequence and another one is sequence similarity that quantifies the sequential nature. Sequence similarity is denoted as the order of occurrence of item sets within two sequences. Length of Longest Common Subsequence (LLCS) with respect to the length of the longest sequence defines the sequence similarity aspect across two sequences. For two sequences $A$ and $B$, sequence similarity is given in Eq. (1).

$$
\operatorname{SeqSim}(A, B)=\frac{\operatorname{LLCS}(A, B)}{\max (|A|,|B|)}
$$

Set similarity is also known as Jaccard similarity measure, which is defined as ratio of the number of common item sets and the number of unique item sets in two sequences. Thus, for two sequences $A$ and $B$, set similarity is given Eq. (2).

$$
\operatorname{SetSim}(A, B)=\frac{|A \cap B|}{|A \cup B|}
$$

Thus, S3M is constituted as a function of both set similarity and sequence similarity and is defined in Eq. (3).

$$
S^{3} M(A, B)=P \times \frac{\operatorname{LLCS}(A, B)}{\max (|A|,|B|)}+q \times \frac{|A \cap B|}{|A \cup B|}
$$

Where, $p+q=1, p, q \geq 0$.

Here, $p$ and $q$ is represented as the relative weights. Once the similarity between the web users are computed, the initial set of the clusters is formed using PFCM.

\subsection{Possibilistic fuzzy c-means}

The PFCM is a data clustering technique, where each data point is a part of the cluster to a level 
indicated by the membership grade. In the clustering module, PFCM is dependent on the reduction of the objective function, which is illustrated in the following Eq. (4).

$$
\begin{aligned}
& J_{P F C M}(U, T, V)=\sum_{i=1}^{C} \sum_{j=1}^{n}\left(u_{i j}^{m}+\right. \\
& \left.t^{n}\right) d^{2}\left(x_{j}, v_{i}\right)
\end{aligned}
$$

With the following constraints:

$$
\begin{aligned}
& \sum_{i=1}^{c} \mu_{i j}=1, \forall j \in\{1, \ldots, n\} \\
& \sum_{j=1}^{n} t_{i j}=1, \forall i \in\{1, \ldots, c\}
\end{aligned}
$$

Where, $J_{P F C M}$ is the objective function, $U$ is represented as the partition matrix, $T$ is represented as the typicality matrix, $V$ is denoted as the vector of cluster centres.

The outcome of the objective function is achieved using an iterative approach, where the degree of membership and the cluster centres are mathematically represented in Eqs. (7), (8) and (9).

$$
\begin{gathered}
\mu_{i j}=\left[\sum_{k=1}^{c}\left(\frac{d x_{j}, v_{i}}{d x_{j}, v_{k}}\right)^{\frac{2}{m-1}}\right]^{-1}, 1 \leq i \leq c, 1 \leq j \leq n \\
t_{i j}=\left[\sum_{k=1}^{n}\left(\frac{d x_{j}, v_{i}}{d x_{j}, v_{k}}\right)^{\frac{2}{n-1}}\right]^{-1}, 1 \leq i \leq c, 1 \leq j \leq n \\
v_{i}=\frac{\sum_{k=1}^{n}\left(u_{i k}^{m}+t_{i k}^{n}\right) x_{k}}{\sum_{k=1}^{n}\left(u_{i k}^{m}+t_{i k}^{n}\right)}, 1 \leq i \leq c
\end{gathered}
$$

Where, $n$ is represented as the number of data points, $c$ is represented as the number of cluster centers, which are described by the coordinates $\left(x_{j}, v_{i}\right)$ and it is used to calculate the distance between cluster center and data sets.

PFCM constructs possibilities and memberships with normal prototypes and cluster centers for every cluster. Choosing the objective function is the important aspect for the performance of cluster methodology for accomplishing better clustering. Whereas, the clustering performance is based on the objective function, which is utilized for clustering. For developing an effective objective function, the following set of requirements are considered.

- Distance between the clusters should be reduced.

- Distance between the data points, which allocated in the clusters should be reduced.

The desirability between the clusters and data are modelled by the objective function. Further, the objective function of PFCM is improved by using driven prototype learning of parameter $\alpha$. The learning procedure $\alpha$ is a dependent exponential separation strength between the clusters and it is updated at every iteration. The parameter $\alpha$ is represented in Eq. (10).

$$
\alpha=\exp \left(-\min _{i \neq k} \frac{\left\|v_{i}-v_{k}\right\|^{2}}{\beta}\right)
$$

Where, $\beta$ is represented as sample variance, mathematically, it is represented in Eq. (11).

$$
\begin{aligned}
& \beta=\frac{\sum_{j=1}^{n}\left\|x_{j}-\bar{x}\right\|^{2}}{n} \\
\text { Where, } \quad \bar{x} & =\frac{\sum_{j=n}^{n} x_{j}}{n}
\end{aligned}
$$

Then, a weight parameter is introduced to calculate the common value of $\alpha$. Each point of the database consists of a weight in relationship with each cluster. So, the usage of weight function delivers a better classification outcome, especially in the case of noise data. The general equation of weight function is determined in Eq. (12).

$$
w_{j i}=\exp \left(-\frac{\left\|x_{j-} v_{i}\right\|^{2}}{\left(\sum_{j=1}^{n}\left\|x_{j-} \bar{v}\right\|^{2}\right) \times c / n}\right)
$$

Where, $w_{j i}$ is denoted as the weight function of the point $j$ with the class $i$. The step by step procedure of PFCM is represented in Fig. 2, and also effectively explained below,

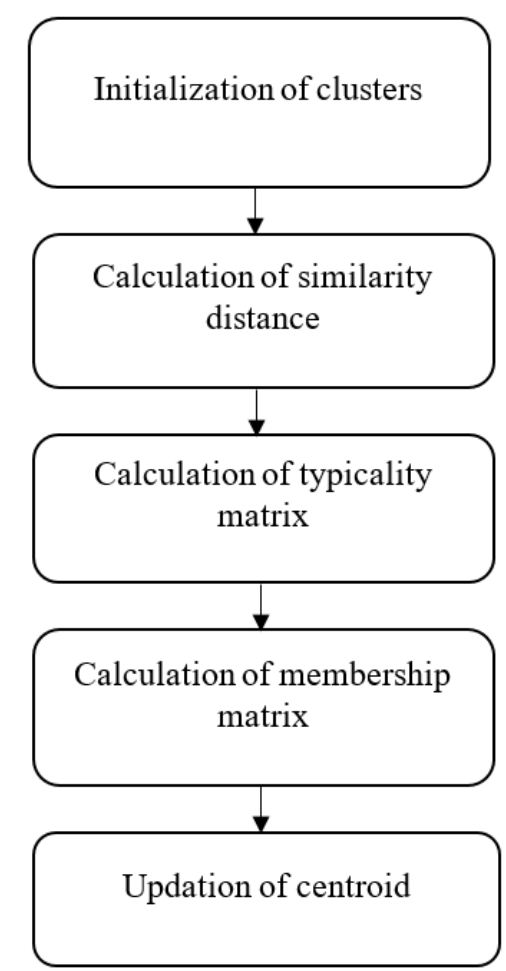

Figure.2 Step by step procedure of PFCM 
- Initialization: At first, the number of clusters is furnished by the user, which are identical with respect to every segment.

- Calculation of similarity distance: When the number of clusters is determined, the evaluation of the distance between the centroids and data points for each segment is carried out.

- Calculation of typicality matrix: After the estimation of distance matrix, the typicality matrix is evaluated, which is obtained from the PFCM.

- Calculation of membership matrix: The evaluation of the membership matrix $M_{i k}$ is performed by means of assessing the membership value of data point, which is gathered from the PFCM.

- Updation of centroid: After the generation of clusters, the modernization of the centroids is updated.

The relative procedure is performed again and again till the modernized centroids of each and every cluster becomes identical in successive iterations. To improve the clustering efficiency of the proposed method, a new association rule mining technique is developed after the clustering algorithm and it is discussed in the below section.

\subsection{Rapid frequent pattern growth}

In this section, the RFPG algorithm is examined on the hypothetical databases for recommending the related web-pages. Initially, the RFPG algorithm has a node-link field and a header table for maintaining the connection between the nodes, whose web-pages are equal in the tree. If the RFPG algorithm does not have such structure means, it is a simpler prefix tree. Then, each node in the RFPG tree has the successor and predecessor fields, if the nodes in the RFPG trees are none. The predecessor node is determined by the predecessor traversal of the RFPG tree.

In the predecessor traversal, a node $\mathrm{M}$ is assigned to the predecessor rank, before all the predecessor children's are traversed from left to right. Also, the predecessor measures the time, when node $M$ is assessed during the predecessor traversal. Likewise, the successor nodes are determined by using successor traversal of the tree. In the successor traversal, from the root node the consecutive successor children traverse from left to right, according to the ranking of frequent web pages. At last, after building the RFPG tree, it is utilized for frequent pattern mining, which is the complex and recursive procedure. However, RFPG tree is utilized for generating the predecessor and successor code of each node. Later, the related web-pages are identified by matching the traversal of the web log using user query, then the RFPG tree finishes the whole task and terminate.

The step by step procedure of RFPG tree algorithm is determined below.

\section{Algorithm (RFPG-construction)}

Input: MSNBC dataset, and castings technology international dataset

Output: RFPG-tree (related web-pages based on user request)

Method: Construction of RFPG-tree

Generate frequent patterns

1) Initially, scan the databases and convert the web pages into number of sequences (S). Then, sort the sequences (S) into descending order as (d), which contains a list of frequent web pages.

Construction of RFPG-tree

2) Create a root for RFPG-tree, label it as "null". Scan the databases again, for each iteration and arrange the frequent webpages into descending order (d), then insert the frequent web-pages into RFPGtree. node

Generate predecessor and successor for each

3) Scan RFPG-tree by predecessor traversal to generate the predecessor code. Scan RFPG-tree again by successor traversal to generate the successor code.

\section{Experimental outcome}

For experimental simulation, Net-Beans (version 6.2) was employed on $\mathrm{PC}$ with $3.2 \mathrm{GHz}$ with i5 processor. In order to estimate the efficiency of the proposed algorithm, the performance of the proposed method was compared with Singular Value Decomposition (SVD) [18] on the reputed datasets: MSNBC dataset, and castings technology international dataset. The performance of the proposed methodology was compared in terms of accuracy and precision.

\subsection{Performance measure}

The relationship between the input and output variables of a system understand by employing the suitable performance metric like precision. The general formula for calculating the precision of the personalized web page recommendation system is given in Eq. (13) as.

$$
\text { Precision }=\frac{T P}{T P+F P} \times 100
$$


Table 1. Accuracy comparison of existing and proposed method using MSNBC dataset

\begin{tabular}{|c|c|c|c|c|c|c|c|}
\hline Methods & $\begin{array}{c}\text { No. of } \\
\text { predictions }\end{array}$ & $\begin{array}{c}\text { Random } \\
(\mathbf{\%})\end{array}$ & $\begin{array}{c}\text { Sample } \\
\mathbf{1}(\boldsymbol{\%})\end{array}$ & $\begin{array}{c}\text { Sample } \\
\mathbf{2 ( \% )}\end{array}$ & $\begin{array}{c}\text { Sample } \\
\mathbf{3 ( \% )}\end{array}$ & $\begin{array}{c}\text { Sample } \\
\mathbf{4 ( \% )}\end{array}$ & $\begin{array}{c}\text { Sample } \\
\mathbf{5 ( \% )}\end{array}$ \\
\hline \multirow{3}{*}{ SVD } & 1 & 5.88 & 11.76 & 12.50 & 9.38 & 17.24 & 13.64 \\
\cline { 2 - 8 }$[18]$ & 2 & 11.76 & 23.53 & 18.75 & 18.75 & 24.14 & 13.64 \\
\cline { 2 - 8 } & 3 & 17.65 & 29.41 & 37.50 & 28.13 & 31.03 & 22.73 \\
\hline PFCM- & 1 & 18.23 & 22.54 & 18.13 & 27.3 & 29.6 & 27.5 \\
\cline { 2 - 8 } RFPG & 2 & 45.7 & 53.21 & 43.6 & 58.7 & 61.6 & 58.7 \\
\cline { 2 - 8 } & 3 & 77 & 76.21 & 72.6 & 83 & 89 & 87 \\
\hline
\end{tabular}

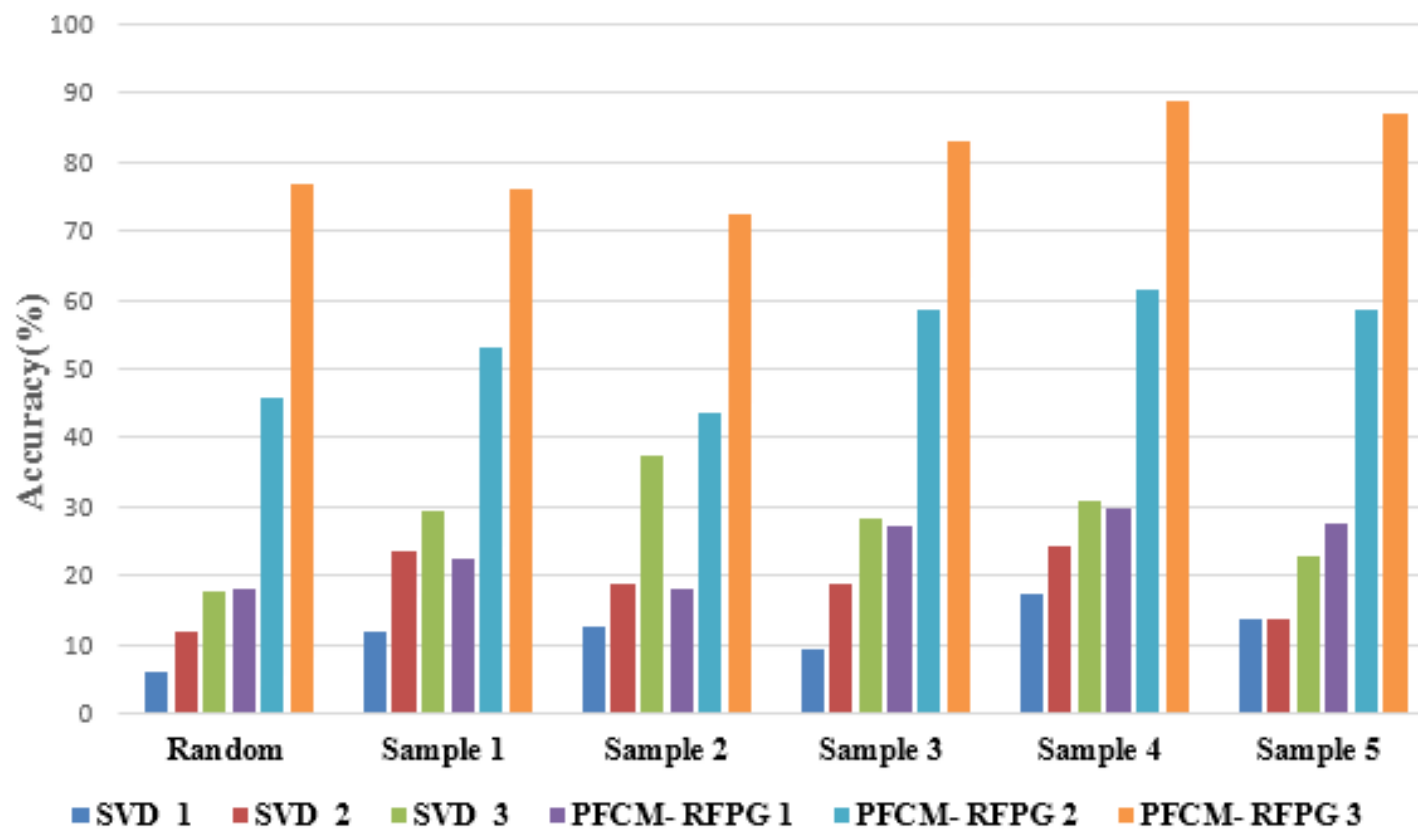

Figure. 3 Performance comparison using MSNBC dataset

Accuracy is the measure of statistical variability and a description of random errors. The general formula of accuracy for determining personalized web page recommendation system is given in Eq. (14).

$$
\text { Accuracy }=\frac{T P+T N}{T P+T N+F P+F N} \times 100
$$

Where, $T P$ is represented as true positive, $F P$ is denoted as a false negative, TN is represented as true negative and $\mathrm{FN}$ is stated as a false negative

\subsection{Result for MSNBC dataset}

In this experimental analysis, MSNBC dataset is assessed for comparing the performance evaluation of existing method and the proposed scheme. In table 1 , the accuracy of the proposed and existing methodology is compared to the five samples and three predictions. The average accuracy of existing methods (SVD) for each prediction delivers $11.7334 \%, 18.42 \%$, and $27.74 \%$. Likewise, the average accuracy of the proposed method (PFCM-
RFPG) for each prediction delivers $23.884 \%$, $53.585 \%$, and $80.80 \%$. The performance evaluation of MSNBC dataset is denoted in Fig. 3.

\subsection{Result for castings technology international dataset}

The experimental analysis of castings technology international dataset is evaluated for comparing the performance of existing and the proposed method. In table 2, the accuracy of the proposed and existing methodology is also compared to five samples and three predictions. The average accuracy of existing methods (SVD) for each prediction delivers $7.181 \%$, $27.24 \%$, and $32.17 \%$. Similarly, the average accuracy of the proposed method (PFCM- RFPG) for each prediction delivers $21.08 \%, 53.53 \%$, and $93.16 \%$. The table 1 and 2 confirmed that the proposed approach performed effectively compared to the existing methods on the both MSNBC dataset and castings technology international dataset. The performance evaluation of castings technology international dataset is presented in Fig. 4. 
Table 2. Accuracy comparison of existing and proposed method using castings technology international dataset

\begin{tabular}{|c|c|c|c|c|c|c|c|}
\hline \multirow{2}{*}{ Methods } & $\begin{array}{c}\text { No. of } \\
\text { predictions }\end{array}$ & $\begin{array}{c}\text { Random } \\
(\mathbf{\%})\end{array}$ & $\begin{array}{c}\text { Sample 1 } \\
(\mathbf{\%})\end{array}$ & $\begin{array}{c}\text { Sample 2 } \\
(\boldsymbol{\%})\end{array}$ & $\begin{array}{c}\text { Sample 3 } \\
(\boldsymbol{\%})\end{array}$ & $\begin{array}{c}\text { Sample 4 } \\
(\boldsymbol{\%})\end{array}$ & $\begin{array}{c}\text { Sample 5 } \\
(\boldsymbol{\%})\end{array}$ \\
\hline \multirow{3}{*}{ SVD[18] } & 1 & 6.25 & 9.38 & 6.90 & 7.41 & 6.90 & 6.25 \\
\cline { 2 - 8 } & 2 & 12.50 & 21.88 & 31.03 & 18.52 & 48.28 & 31.25 \\
\cline { 2 - 8 } & 3 & 18.75 & 28.13 & 34.48 & 25.93 & 48.28 & 37.50 \\
\hline \multirow{2}{*}{$\begin{array}{c}\text { PFCM- } \\
\text { RFPG }\end{array}$} & 1 & 19.8 & 22 & 20.4 & 19.3 & 24 & 21 \\
\cline { 2 - 8 } & 2 & 50.6 & 53.5 & 57.8 & 49.6 & 57.4 & 52.3 \\
\hline
\end{tabular}

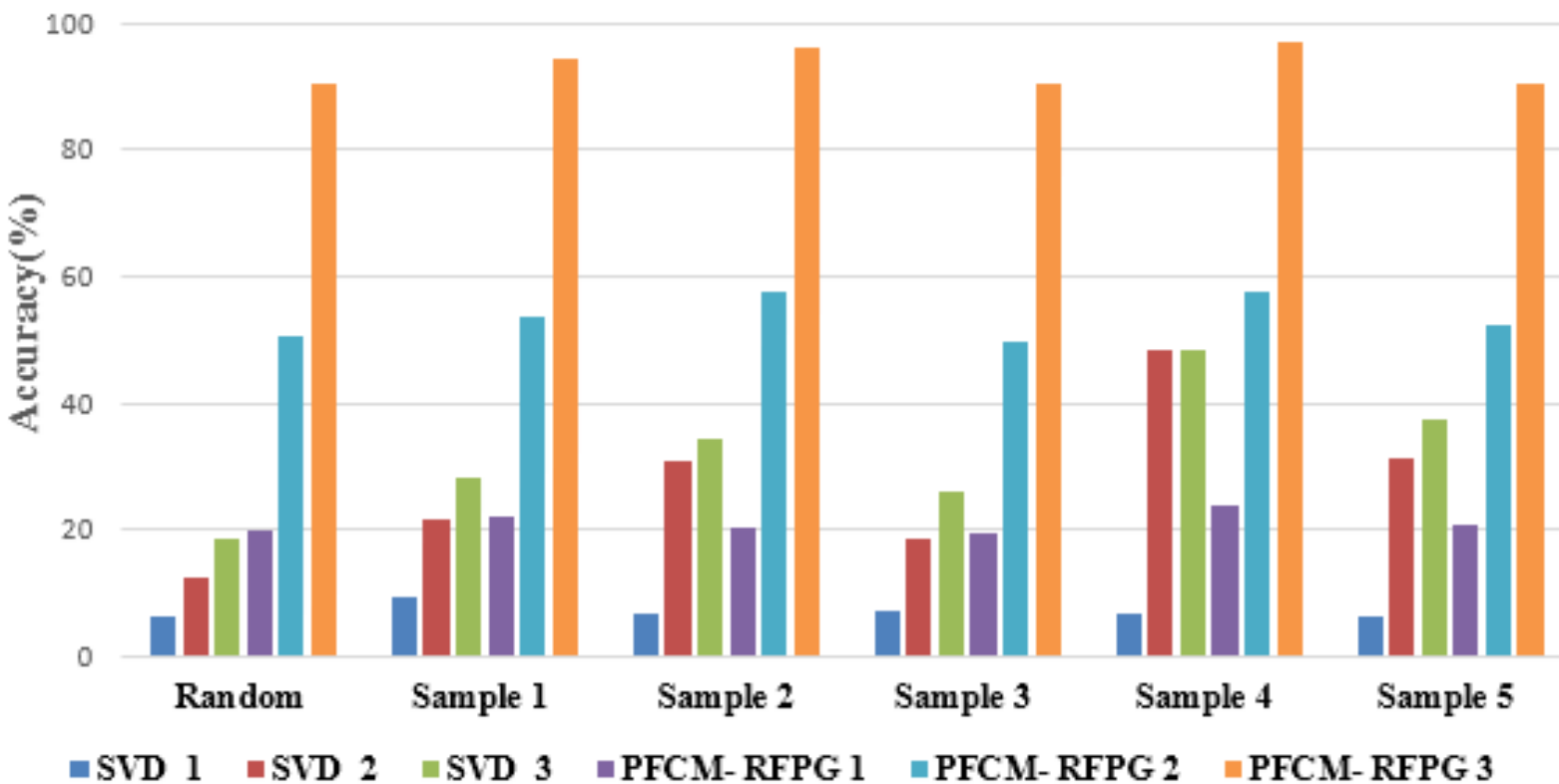

Figure.4 Performance comparison using castings technology international dataset

Table 3 presented the performance of various clustering (M) using PFCM- RFPG approach for improving the personalized web page recommendation system. Table 3 clearly shows that the PFCM- RFPG approach improves the precision in web page recommendation system up to $50 \%$ compared to the existing method in the both MSNBC dataset and castings technology international dataset. R. Mishra, P. Kumar, and B. Bhasker [18] proposed a new recommendation system using similarity upper approximation and SVD for generating the recommendations for users. The graphical representation of precision comparison is represented in Figs. 5 and 6.

Table 3. Precision comparison of existing and proposed method

\begin{tabular}{|c|c|c|c|c|c|c|c|}
\hline Methods & \multicolumn{4}{|c|}{ MSNBC dataset } & \multicolumn{3}{|c|}{$\begin{array}{c}\text { Castings technology international } \\
\text { dataset }\end{array}$} \\
\hline \multirow{5}{*}{ SVD [18] } & Trails & $\begin{array}{c}\text { First } \\
(\%)\end{array}$ & $\begin{array}{c}\text { Second } \\
(\%)\end{array}$ & $\begin{array}{c}\text { Third } \\
(\%)\end{array}$ & $\begin{array}{r}\text { First } \\
(\%)\end{array}$ & $\begin{array}{c}\text { Second } \\
(\%)\end{array}$ & $\begin{array}{c}\text { Third } \\
(\%)\end{array}$ \\
\hline & $\mathrm{M}=20$ & 12.61 & 19.83 & 28.12 & 7.37 & 30.19 & 34.86 \\
\hline & $M=22$ & 10.60 & 16.74 & 24.38 & 7.98 & 21.00 & 27.51 \\
\hline & $M=32$ & 11.02 & 17.86 & 28.80 & 8.20 & 23.94 & 24.89 \\
\hline & Random & 5.88 & 11.76 & 17.65 & 4 & 8 & 12 \\
\hline \multirow{4}{*}{$\begin{array}{c}\text { PFCM- } \\
\text { RFPG }\end{array}$} & $\mathrm{M}=20$ & 23.88 & 53.58 & 80.8 & 21.8 & 53.53 & 93.17 \\
\hline & $M=22$ & 20.21 & 51.56 & 81.23 & 21.6 & 52 & 90.25 \\
\hline & $M=32$ & 20.75 & 51.7 & 81.9 & 22.3 & 51.7 & 91.3 \\
\hline & Random & 22.7 & 52.35 & 81.6 & 21.15 & 51.32 & 90.36 \\
\hline
\end{tabular}




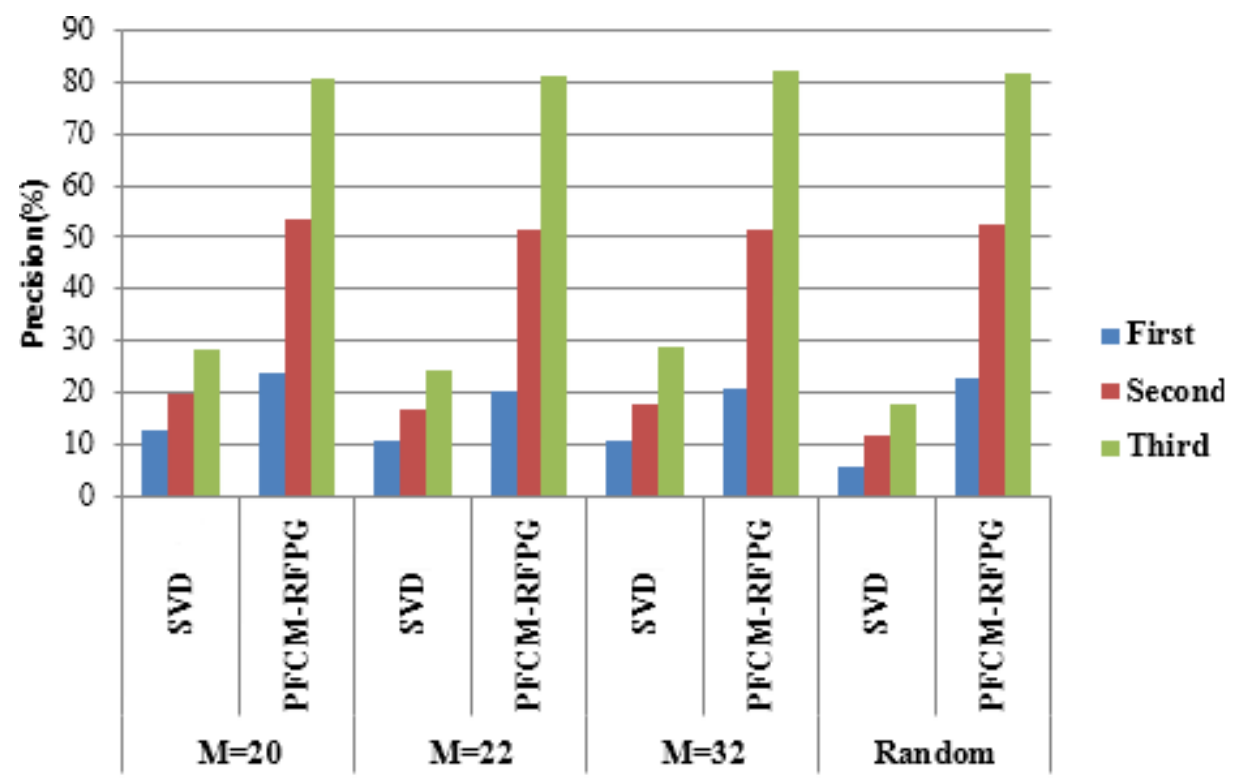

Figure.5 Precision comparison for MSNBC dataset

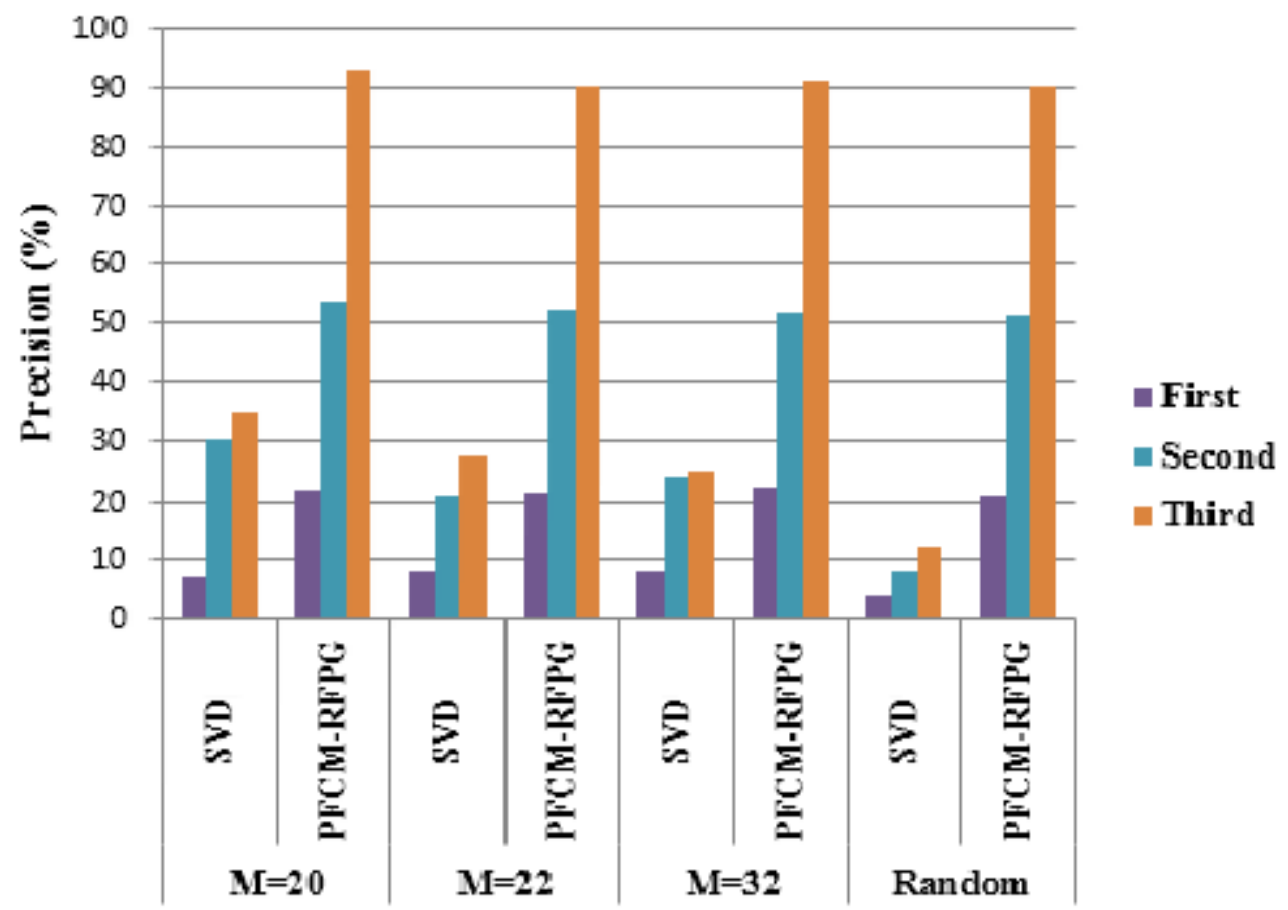

Figure.6 Precision comparison for castings technology international dataset

\section{Conclusion}

In this experimental research, an effective personalized web page recommendation system is proposed using PFCM-RFPG scheme for recommending the related web pages to the user. The PFCM method is used as a clustering frame-work for identifying the neighbourhood of each user profile. After identifying the neighbourhood profile, the RFPG algorithm is employed for mining the frequent web pages. In this experimental analysis, the proposed approach obtained 10-50\% of improvement in precision and 50-60\% of improvement in accuracy of web page recommendation for MSNBC and castings technology international dataset, respectively. While comparing with existing work, the proposed approach showed effective results in terms of accuracy and precision. The web page recommendation accuracy is more significant in PFCM-RFPG than the previous methodologies. In the future work, for further improving the web page recommendation accuracy, we will develop a new association rule mining approach with an adaptive clustering methodology. 


\section{References}

[1] A. Hawalah and M. Fasli, "Dynamic user profiles for web personalisation", Expert Systems with Applications, Vol.42, No.5, pp.2547-2569, 2015.

[2] R. Duwairi and H. Ammari, "An enhanced CBAR algorithm for improving recommendation systems accuracy", Simulation Modelling Practice and Theory, Vol.60, pp.54-68, 2016.

[3] G. Castellano, A.M. Fanelli, and M.A. Torsello, "NEWER: A system for NEuro-fuzzy WEb Recommendation", Applied Soft Computing, Vol.11, No.1, pp.793-806, 2011.

[4] M. Göksedef and Ş. Gündüz-Öğüdücü, "Combination of Web page recommender systems", Expert Systems with Applications, Vol.37, No.4, pp.2911-2922, 2010.

[5] J. Martinez-Romo and L. Araujo, "Updating broken web links: An automatic recommendation system", Information Processing \& Management, Vol.48, No.2, pp.183-203, 2012.

[6] H. Wen, L. Fang, and L. Guan, "A hybrid approach for personalized recommendation of news on the Web", Expert Systems with Applications, Vol.39, No.5, pp.5806-5814, 2012.

[7] M. Jalali, N. Mustapha, M.N. Sulaiman, and A. Mamat, "WebPUM: A Web-based recommendation system to predict user future movements", Expert Systems with Applications, Vol.37, No.9, pp.6201-6212, 2010.

[8] J. Jooa, S. Bangb, and G. Parka, "Implementation of a Recommendation System Using Association Rules and Collaborative Filtering", Procedia Computer Science, Vol.91, pp.944-952, 2016.

[9] S. Abrishami, M. Naghibzadeh, and M. Jalali, "Web page recommendation based on semantic web usage mining", Social Informatics, pp.393405, 2012.

[10] M. Pannu, R. Anane, M. Odetayo, and A. James, "Explicit user profiles in web search personalisation," In: Proc. of $15^{\text {th }}$ International Conf. on Computer Supported Cooperative Work in Design (CSCWD), pp.416-421, 2011.

[11] T.T.S. Nguyen, H.Y. Lu, and J. Lu, "Web-page recommendation based on web usage and domain knowledge", IEEE Transactions on Knowledge and Data Engineering, Vol.26, No.10, pp. 2574-2587, 2014.

[12] R. Geetharamani, P. Revathy, and S.G. Jacob, "Prediction of user's webpage access behaviour using association rule mining", Sadhana, Vol.40, No.8, pp.2353-2365, 2015.

[13] R. Katarya and O.P. Verma, "An effective web page recommender system with fuzzy c-mean clustering", Multimedia Tools and Applications, Vol.76, No.20, pp.21481-21496, 2017.

[14] N.T.S.H. Rizvi, and R.R. Keole, "Web page recommendation in information retrieval using domain knowledge and web usage mining", International Journal of Science, Engineering and Technology Research, Vol.4., No.5, 2015.

[15] S.P. Malarvizhi and B. Sathiyabhama, "Frequent page sets from web log by enhanced weighted association rule mining", Cluster Computing, Vol.19, no.1, pp. 269-277, 2016.

[16] A.S. Tewari and A.G. Barman, "Collaborative Recommendation System Using Dynamic Content based Filtering, Association Rule Mining and Opinion Mining”, International Journal of Intelligent Engineering and Systems, Vol.10, No.5, pp.75-66, 2017.

[17] V. Vellaichamy and V. Kalimuthu, "Hybrid Collaborative Movie Recommender System Using Clustering and Bat Optimization", International Journal of Intelligent Engineering and Systems, Vol.10, No.5, pp.38-47, 2017.

[18] R. Mishra, P. Kumar, and B. Bhasker, "A web recommendation system considering sequential information", Decision Support Systems, Vol.75, pp.1-10, 2015. 